\title{
Naissance de la Biospéologie
}

Par Constantin Motas, Bucarest ${ }^{1}$ )

Avec 2 portraits

La découverte, en 1904, dans une des grottes du Drach (île de Majorque) du minuscule Cirolanide Typhlocirolana Moraguesi, qu'il décrivit une année après, fut pour E. G. Racovitza comme la fameuse pomme de Newton: un rayon lumineux qui éclaira tout d'un coup la voie qu'il allait définitivement suivre.

Par une intuition quasi géniale il comprit immédiatement l'importance que peuvent présenter pour le problème de l'évolution les êtres cavernicoles qui, dans leur organisation, portent l'empreinte si puissante du milieu souterrain.

Il abandonna pour toujours les recherches océanographiques où il avait été initié par son maître et ami G. Pruvot et, à partir de 1905, il se mit à fouiller les grottes.

A ce changement complet d'orientation scientifique - ainsi qu'il le déclare lui-même - contribua pour une large part le fondateur de la Spéologie moderne, E.-A. Martel, par ses passionnants récits sur les beautés inouies du monde souterrain.

En s'adonnant dorénavant corps et âme à la recherche méthodique des grottes, il ramassa un important matériel biospéologique et des observations dont il allait forger la science nouvelle du monde vivant souterrain.

Ainsi naquit, après une gestation de trois ans, la Biospéologie. Le 15 mai 1907 est en effet le jour de la naissance de celle-ci, lorsque Racovitza fit paraître dans les «Archives de Zoologie expérimentale et générale» de Paris, créées par son maitre Henri de Lacaze-Duthiers, son remarquable mémoire intitulé «Essai sur les problèmes biospéologiques», véritable condensé, en une centaine de pages à peine, de toute la biologie souterraine.

Il est à remarquer, comme on l'a souvent dit, que malgré les grands progrès que la Biospéologie a fait depuis lors, le «célèbre manifeste

1) Institutul de Speologie «Emil G. Racovitza», Bucuresti 32 . 
de Racovitza», suivant l'expression du Professeur Vandel (1953), plus d'un demi-siècle après sa parution, n'a rien perdu ni de sa valeur, ni de son actualité.

Cet «Essai» fut considéré par Antipa (1926) comme le certificat de naissance de la Biospéologie et par le Docteur R. Jeannel $(1948,1950)$ comme le statut fondamental de la Biospéologie.

Dans ce classique mémoire, œuvre de sa jeunesse, Racovitza posa clairement tous les problèmes que soulève la vie dans le milieu souterrain en toute leur complexité. Il en résoudra quelques-uns, en signalant en même temps les écueils à éviter pour résoudre les autres. Il indiqua la direction qu'il fallait donner aux recherches futures, en traçant le programme à suivre et qu'il suivit lui-même sans se lasser.

Racovitza fut ainsi amené à envisager, dans son ensemble, le domaine souterrain qu' il divisa en : $1^{\circ}$-Grottes accessibles à l'homme, $2^{\circ}$-fentes inaccessibles à l'homme, $3^{\circ}$-nappes phréatiques, $4^{\circ}$-habitat hypogé, $5^{\circ}$-microcavernes et $6^{\circ}$-cavernes artificielles.

J'ai montré ailleurs (1962) que différents auteurs tels que Cuénot (1932), Leruth (1939), Chappuis (1950), Husson (1936, 1960) ont plus ou moins modifié en simplifiant ou en amplifiant le système proposé par Racovitza. Je prie le lecteur de vouloir bien s'y reporter.

Qu'on me permette de dire un mot seulement sur l'étendue du domaine souterrain.

Suivant l'avis de Racovitza (1907) et de Jeannel (1937), la surface que le domaine souterrain offre à ses habitants serait égale à celle du domaine alpestre et du domaine désertique réunies. Mais si l'on voulait se reporter seulement à l'immense étendue de la nappe phréatique générale, résultant du drainage des continents voisins par l'océan, l'on arriverait à la conclusion que le domaine souterrain est infiniment plus vaste que ne le pensaient Racovitza et Jeannel.

Après la découverte du Typhlocirolana Moraguesi dans la «Cueva del Drach», Racovitza dépouilla toute une bibliographie relative au domaine souterrain et aux habitants de ce vaste monde. Il en sortit complètement deçu. Car partout régnait «un chaos inextricable de faits, de théories et d'erreurs».

L'écologie, l'éthologie, la biogéographie, l'évolution des cavernicoles étaient encore à l'état nébuleux. Le champ était encombré de tout un fatras d'observations contradictoires, de suppositions inexactes voire même complètement fausses ou fantaisistes, d'expériences à peine ébauchées ou mal interprétées.

Il était donc grand temps de déblayer le terrain de ces matériaux inutiles sinon nuisibles, afin de pouvoir élever plus facilement l'édifice 
de la Biospéologie avec des pierres solides qui puissent résister à toute épreuve.

Ce fut le grand mérite de Racovitza de mettre de l'ordre dans ce chaos, de débarrasser le terrain de ce matériel informe et encombrant, et de construire ensuite la charpente entière, l'ossature puissante de la nouvelle science du monde vivant souterrain.

Mais l'édifice n'aurait pu ètre élevé sans l'apport architectonique de l'œuvre considérable de son collaborateur et ami, le Docteur René Jeannel, le grand entomologiste français. C'est avec lui que Racovitza fonda chez nous en 1920, à Cluj (Klausenburg, Kolozsvar), le premier Institut de Spéologie ${ }^{2}$ du monde et dont Monsieur le Professeur Jeannel fut le premier sous-directeur. Ils ont fondé "Biospeologica» étude de l'histoire naturelle du domaine souterrain; ils ont publié en collaboration le cycle monumental «Enumération des grottes visitées», ouvrage en plusieurs volumes connu dans le monde entier. Ils sont, coïncidence étrange, entrés pour la première fois dans une grotte, à la même époque: Racovitza, en 1904, dans la Cueva del Drach, Jeannel, la même année, dans la grotte d'Oxibar près de Camou-Cihigue dans les BassesPyrénées. Les vies de ces deux grands naturalistes sont parallèles, à telle enseigne, qu'ils ont été aussi tous les deux attirés par les solitudes mystérieuses des régions antarctiques. En effet, Racovitza participa comme naturaliste au voyage du glorieux «Belgica» à l'Antarctique (1897-1899), Mr. Jeannel à la croisière du «Bougainville» (1938) au seuil de l'Antarctique.

Pour ces raisons le nom de Racovitza et celui du Docteur Jeannel seront à jamais associés.

Mais, vouloir montrer ici la part de chacun des deux maîtres à l'architectonique de la Biospéologie est une entreprise bien difficile sinon impossible.

Voici pourquoi nous nous contenterons d'énumérer les problèmes qui ont préoccupé et passionné Racovitza, et qui devaient préoccuper et passionner aussi son adepte le plus attaché, Mr. Jeannel.

Ce sont: l'influence des facteurs du milieu tels que l'obscurité, la température, l'humidité, la qualité de l'air et de l'eau, le régime alimentaire, etc., sur la vie et l'organisation des cavernicoles, et les réactions de ceux-ci: dépigmentation du tégument, atrophie progressive de l'appareil visuel, développement des organes compensateurs, etc.

2) Huit ans avant la fondation de cet institut, le Dr. C. N. Ionesco (1912, 1913) un élève d'Ernst Haeckel à Jena, après avoir entrepris des recherches spéologiques méthodiques sur les grottes de l'Olténie (Roumanie), publia les premiers résultats de ses recherches qui constituent le début des études biospéologiques dans notre pays. 
Racovitza (1907) a insisté assez longuement aussi sur: le peuplement du domaine souterrain et son ancienneté, sur les moyens de colonisation de ce domaine, sur le rôle de la lutte pour l'existence et de la sélection naturelle.

Vu l'espace restreint mis à notre disposition, nous ne pouvons pas nous appesantir sur ces problèmes variés, dont la complexité a été très bien mise en relief par Racovitza ainsi que par Mr. Jeannel (1943, 1950).

Le premier a, dans son «Essai», non seulement discuté, souvent très amplement les opinions des différents auteurs (Carpenter, Chilton, Darwin, Eigenmann, Garman, Hamann, Lankester, Packard, Viré etc.) sur ces problèmes, en battant en brêche les plus biscornues, mais encore «ce grand semeur d'idées» - comme vient de le qualifier récemment Delamare Deboutteville (1960) - a semé, tant dans ce mémoire que dans toute son œuvre, des idées «toujours originales, parfois même révolutionnaires», suivant Guiart et Jeannel (1948), «qui font de l'œuvre de Racovitza, celle d'un très grand naturaliste".

Il s'est élevé (1907) même contre Darwin (1859) et Packard (1889, 1894) qui ont nié l'existence de la sélection naturelle dans le domaine souterrain, et par conséquent l'action de celle-ci sur les animaux qui $\mathrm{y}$ vivent. On sait qu'ils ont soutenu que chez les animaux vivant dans l'obscurité l'absence de l'œil est due au non-usage. Hamann (1896) lui ne croyait pas à une obscurité totale dans les grottes; il pensait que les cavernicoles dérivent de formes aux yeux déjà en état d'atrophie. Tout en étant néolamarckien, Racovitza (1907, p. 454) rejettera cette interprétation lamarckienne trop simpliste et fort curieuse chez Darwin. Il est à remarquer que Caullery (1931, p. 269) abondait dans le même sens, déclarant que l'usage ou le non-usage ne sont pas la condition nécessaire et suffisante d'hypertrophie ou d'atrophie des organes.

D'ailleurs, avant Caullery, Mr. Jeannel (1930, p. 78) écrivait: «chez la plus grande partie des groupes de cavernicoles, l'effet héréditaire du non-usage est suffisant pour nous rendre compte que suivant de nombreuses générations qui ont vécu dans l'obscurité complète, l'œil s'est atrophié ou a disparu. Mais chez les autres et plus particulièrement chez les insectes, il semble toutefois que cette évolution aurait des causes plus compliquées.»

Un problème parmi les nombreux problèmes biospéologiques qui ont occupé et préoccupé Racovitza et Jeannel est celui de l'isolement géographique ou physiologique. Par leurs recherches sur la faune cavernicole, ils étaient, sans doute, les plus enclins à attribuer à l'isolement un rôle important dans la formation des variétés ou des espèces. Il est vrai que l'«Essai» (p. 475) ne contient qu'une vague allusion au rôle 
joué par ce facteur. Mais, quelques années après, Racovitza donna de l'espèce une définition de nuance fortement ségrégationniste: «L'espèce est une colonie isolée de consanguins.» Cette idée, chose curieuse, aujourd'hui est adoptée par certains auteurs américains tels que E. Mayr et G. G. Simpson, comme l'a remarqué Vandel (1952, p. 62).

Plus tard, Racovitza (1929), arrivera à la conviction que «'isolement est une condition nécessaire et suffisante pour produire la spéciation", en devenant ainsi l'adepte de Wagner et de Conta - comme nous l'avons montré (Motas, 1962). Jeannel (1930, pp. 127-136) a fait un exposé très clair du problème de la ségrégation en montrant la complexité. Il reviendra (1942, pp. 1-16 et 1943, pp. 99-104) avec de plus amples détails sur l'isolement auquel il attribue lui aussi un rôle très important dans la spéciation: «Il n'est pas douteux que le facteur isolement suffit à lui seul pour déterminer des divergences et, par conséquent des espèces."

Caullery (1931, pp. 352-353) avait déjà signalé que par leurs recherches Racovitza et Jeannel ont apporté de très nombreux faits à l'appui de la ségrégation: «L'isolement des animaux vraiment cavernicoles est absolu dans chaque système de grottes autonome et l'étude détaillée d'un groupe montre que des grottes très voisines mais indépendantes, sont habitées par des espèces distinctes, tandis que celles faisant partie d'un même ensemble et communiquant facilement ont les mêmes habitants.»

La Biospéologie prouve que dans chaque grotte indépendante, isolée, se sont différenciées des formes spéciales qui, le plus souvent, sont des relictes des époques révolues, de véritables "fossiles vivants».

Suivant Racovitza (1907, p. 381), après avoir étudié le plus complètement possible un petit groupe de cavernicoles, il faut procéder à des expériences rigoureuses. La cible de ses flèches ironiques furent les expériences faites par Armand Viré, dans le premier laboratoire souterrain du monde qu'il créa à Paris dans les Catacombes du Muséum d'Histoire Naturelle sous l'égide de A. Milne-Edwards.

Jeannel (1950, p. 16) a rompu aussi beaucoup de lances avec Viré dont «les résultats expérimentaux - dit-il - ne sont pas moins étranges que ses observations anatomiques». Bref, conclut-il, «dans l'histoire de la biospéologie française, A. Viré a été un épisode néfaste».

En effet, Viré a dû commettre de nombreuses et peut-être de lamentables erreurs dans les expériences qu'il a entreprises dans son laboratoire de fortune installé dans une catacombe, et non pas dans une caverne véritable. Mais nous croyons que les critiques de Racovitza et Jeannel ont dépassé leurs pensées et que Viré n'a pas mérité leurs sarcasmes. 
Sans doute Armand Viré a été un spéologue intrépide, courageux et passionné qui risqua maintes fois sa vie, en descendant dans le fond des gouffres pour y chercher des animaux cavernicoles.

Il a été initié aux explorations souterraines par Edmond Renault, en 1894 et 1895, dans les grottes du Jura. Martel, - accompagné dans ses investigations par son dévoué Louis Armand - s'associa, en 1896 et 1895, aussi A. Viré. Sous les auspices du maître celui-ci se voua entièrement aux recherches sur la faune aquatique souterraine.

Le résultat des recherches sur cette faune, poursuivies par lui pendant plusieurs années de suite, fut sa thèse de doctorat-ès-sciences (1899), dédiée à A. Milne-Edwards, et soutenue à la Sorbonne sous la présidence de G. Bonnier.

Certes, c'est un travail de pionnier. Donc, fatalement il contient un grand nombre d'erreurs systématiques, anatomiques, etc. Mais nous pensons que les erreurs de nos devanciers, en nous empêchant de les répéter, contribuent, elles aussi, au progrès de la science. Par conséquent, il ne faut jamais ètre trop sévère avec nos prédécesseurs. D'autant plus que Viré (1904) fut l'inventeur du mot Biospéléologie transformé par Racovitza en celui de Biospéologie - plus court et plus euphonique - et adopté par Jeannel, Chappuis et tant d'autres.

Martel (1919), malgré les âpres critiques de Racovitza et Jeannel, considère Viré comme un de ses "plus fidèles disciples en spéléologie» et auquel on doit d'intéressantes révélations sur la Zoologie souterraine.

\section{RÉSUMÉ}

La Biospéologie est née le 15 mai 1907, lorsque Racovitza fit paraître son remarquable mémoire intitulé: «Essai sur les problèmes biospéologiques» où il a posé, sinon solutionné, tous les problèmes que soulève la vie dans le domaine souterrain. Il y traça le programme des recherches biospéologiques, analysa les conditions d'existence des êtres cavernicoles et leurs influences sur ceux-ci, les modalités de l'évolution des biotes souterrains, leur reproduction, leur distribution géographique, etc...

S'inspirant de la classification proposée par Schiner (1854), il divisa les cavernicoles en trois groupes écologiques : troglobies, troglophiles et trogloxènes, termes adoptés depuis par un très grand nombre de biospéologistes.

Cet «Essai» que Vandel (1953) appelle "le célèbre manifeste de Racovitza» est considéré par Antipa (1926) comme le certificat de naissance de la Biospéologie, et par Jeannel $(1948,1950)$ comme le statut fondamental de celle-ci. Il constitue, en effet, la charpente, l'ossature entière de la science du monde vivant souterrain. Mais l'édifice n'aurait pu être élevé sans l'apport architectonique de l'œuvre considérable de son collaborateur et ami le Docteur 
R. Jeannel. Voilà pourquoi,le nom de Racovitza et celui de Jeannel sont et resteront à jamais unis. Car ils sont les maîtres incontestés de la Biospéologie.

Mais, outre Schiner, en ce qui concerne la classification écologique des cavernicoles, ils ont eu encore un autre précurseur. Je veux nommer A. Viré (1899), spéologue intrépide et passionné qui, accompagnant souvent Martel dans ses explorations souterraines, descendait au fond des gouffres pour y chasser des cavernicoles, souvent au risque de sa vie. Il a étudié la faune souterraine sur laquelle il entreprit des expériences dans le premier laboratoire souterrain du monde, qu'il créa dans les Catacombes du Muséum d'Histoire Naturelle de Paris. Ses expériences ont été à peine ébauchées ou mal interprétées.

Toutefois nous pensons que les critiques qui lui ont été faites par Racovitza et Jeannel sont trop sévères. Et il ne faut pas être trop sévère avec nos prédécesseurs, car leurs erreurs, en nous empêchant de les répéter, contribuent elles aussi au progrès de la Science.

Viré (1904) est l'inventeur du mot Biospéléologie, transformé par Racovitza en Biospéologie, et il fut considéré par Martel (1919) comme le plus fervent de ses disciples en spéléologie.

\section{SUMMARY}

Modern biospeleology dates from May 15, 1907, with the publication of Racovitza's "Essai sur les problèmes biospéologiques." In this paper he posed - if he did not answer - every question raised by life in the subterranean world. He outlined a program of biospeological research, made an analysis of the conditions of existence in the subterranean domain and their influence upon cavernicoles, discussed the evolution of subterranean biota, their geographical distribution, etc. Racovitza modified Schiner's (1854) classification, dividing cavernicoles into troglobites, troglophiles and trogloxenes, terms later adopted by a great number of biospeologists. The «Essai», called "Racovitza's famous manifest» by Vandel, was considered the birth certificate of biospeology by Antipa (1927) and by Jeannel (1948), its fundamental statute.

Jeannel also made major contributions to the young science through his extensive and detailed studies. The names of Racovitza and Jeannel will always be linked as the uncontested masters of biospeology, the founders of Biospeologica, and the authors of «Enumération des grottes visitées».

Apart from Schiner, whose ecological classification of cavernicoles was utilized and modified by Racovitza, they had another forerunner in Viré, a passionate speologist who often accompanied Martel in his subterranean explorations, once meeting with a serious accident in which he was on the brink of death. Viré $(1897,1899)$ studied subterranean faunas, establishing the world's first underground laboratory, where he carried on unsuccessful or ill-interpreted experiments. We consider Racovitza and Jeannel's criticism of him too severe. Let us be more lenient with our forerunners, since their mistakes have also contributed to the progress of science, as well as exempting us from repeating them. 


\section{BIBLIOGRAPHIE}

Antipa, Gr. - 1926: Academia Româna. Discursuri de receptiune, LXI. Discurs de răspuns la primirea lui E. Racovitǎ. Ed. Cultura Natională, Bucaresti, 45-64.

Caullery, M. - 1931: Le problème de l'Evolution. Bibliothèque scient., Payot, Paris, $447 \mathrm{pp}$.

Chappuis, P.A. - 1950: La récolte de la faune souterraine. Notes biosp., V. Edit. Muséum, Paris, 7-35.

Cú́ пот, L. - 1932: La genèse des espèces animales. $3^{e}$ édit., F. Alcan, Paris, I-VIII $822 \mathrm{pp}$.

—, 1936: L'Espèce. Encycl. Scient., G. Doin, Paris, 310 pp.

DARwin, Сн. - 1859: L'origine des espèces au moyen de la sélection naturelle ou la lutte pour l'existence dans la nature. Trad. Ed. Barbier. Libr. C. Reinwald, Paris, $604 \mathrm{pp}$.

Delamare Deboutteville, C. - 1960: Biologie des eaux souterraines littorales et continentales. Actual. scient., industr., 1280, Hermann, Paris, $740 \mathrm{pp}$.

Dudrch, E. - 1932: Biologie der Aggteleker Tropfensteinhöhle „Baradla“ in Ungarn. Verl. Speläologisches Institut, Wien, 246 pp.

Guiart, J., et Jeannel, R. - 1948: Emile-Georges Racovitza (1868-1947) avec un portrait. Arch.Zool. exp. et gén., 86, 1. Introduction par L. Fage. Paris, 1-28.

Hamann, O. - 1896: Europäische Höhlenfauna. Eine Darstellung der in Höhlen lebenden Tierwelt mit besonderer Berücksichtigung der Höhlenfauna Krains, Verl. H. Cortenoble, Jena, 296 pp.

Husson, R. - 1936: Contribution à l'étude de la faune des cavités souterraines artificielles. Ann. Sc. Nat. Zool., XIX, 1-30.

- 1960: Les Crustacés Péracarides des eaux souterraines. Inst. Grand-Ducal de Luxembourg Sect. Sci. natur. phys. et math. "Archives" T. XXVII, Nouv. sér., Luxembourg. 107-119.

Jeannel, R. - 1930: Curs de Biologie generală. Evolutia si Ereditatea. Edit. Universitătii. Instit. Artegrafice «Ardealul», Soc. anon., Cluj, $200 \mathrm{pp}$.

- 1937: Le domaine souterrain. Encycl. franç. V. 4, Paris.

- 1942: La genèse des faunes terrestres. Eléments de biogéographie. Biblioth. Instit. marit. colon. Presses Univ. France, 513 pp.

- 1943: Les fossiles vivants des Cavernes. Gallimard, edit. Paris, 321 pp.

- 1950: Quarante années d'explorations souterraines. Notes biosp., VI, édit. Muséum, Paris, 93 pp.

LERUTh, R. - 1939: La biologie du domaine souterrain et la faune cavernicole de la Belgique. Mus. roy. Hist. natur. Belgique, 87, Bruxelles, 506 pp.

Martel, E. A. - 1919: L'Evolution souterraine. Bibl. Phil. scient. E. Flammarion, Paris, $323 \mathrm{pp}$.

Motas, C. - 1962: Procédé des sondages phréatiques. Division du domaine souterrain. Classification écologique des animaux souterrains. Le psammon. Acta, VIII, 7, Skoplje (Macéd.), 135-173.

- 1962: Emil G. Racovitza: Founder of Biospeology, Bull. Nat. Spel. Soc., 24, 1, Virginia (USA), 1-8.

- 1962 b: Sur les Acariens phréatiques, leur Distribution géographique, leur Origine. Zool.Anz., 168, 7-10, 325-350. 
PACKARD, A. S. - 1889: The cave fauna of North America with remarks on the anatomy of the brain and origin of the blind species. Mem. Nation. Acad. Sci. Washington, 156 pp. Cit. apud Racovitza (1907).

Racovitza, E.-G. - 1907: Essai sur les problèmes biospéologiques. Biospeologica IA Arch.Zool. exp. gén., 4e sér., VI, 15 mai 1907, Paris, 372-488.

- 1912: Cirolanides, Biospeologica XXVII, Arch. Zool. exp. gén. $1^{\text {re }}$ sér., 5. X, Paris, 203-329.

- 1927: Speologia, o stiinta nouǎ a stǎvechilor taine subpǎmăntenesti. Edit. "Astra», 1, Tipogr. "Ardealul», Cluj, 68 pp.

- 1929: Evolutia si problemele ei. Edit. «Astra», 6, Tipogr. «Ardealul», Cluj, $183 \mathrm{pp}$.

Schiner, I. R. - 1854: Fauna der Adelsberger-, Lueger- und Magdalenengrotte. In A. Schmidl: Die Grotten und Höhlen von Adelsberg, Lueg, Planina und Loos. Braunmüller, Wien, 231-272; 316 pag. Cit. apud. Racovitza (1907).

VANDEL, A. - 1952: Etude des Isopodes terrestres récoltés an Vénézuéla par le Dr. G. Marcuzzi. Mem. Mus. Cis. Storia Natur. Verona, III, 59-203.

- 1953: Les recherches biospéologiques en France. Premier Congr. Intern. Spéléol., Paris, 3, 9-12.

Viré, A. - 1899: Essai sur la faune obscuricole de France. Etude particulière de quelques formes zoologiques. Thèse, J. Baillière, Paris, $157 \mathrm{pp}$.

- 1904: La biospéléologie, C.R.Ac.Sci. CXXXIX, Paris, 992-995.

Planche hors-texte: En haut: E. G. Racovitza. En bas: R. Jeannel. 
E.- G. R A C V T Z A



R. JEA N N L L 